\title{
Comparison of Lipid-Lowering Effects of Anagliptin and Miglitol in Patients With Type 2 Diabetes: A Randomized Trial
}

\author{
Takahiro Iijima $^{\text {a, b }}$, Kazutaka Aoki ${ }^{\mathrm{a}, \mathrm{c}}$, Yoshinobu Kondo ${ }^{\mathrm{a}}$, \\ Yasuo Terauchi ${ }^{\mathrm{a}, \mathrm{d}}$
}

\begin{abstract}
Background: Recently, we reported that the level of lathosterol, a cholesterol synthesis marker, was suppressed after 1 month of treatment with anagliptin, a dipeptidyl peptidase- 4 inhibitor. In this study, we administered either anagliptin or miglitol, an alphaglucosidase inhibitor, for 3 months in patients with type 2 diabetes and compared the lipid-lowering effects of anagliptin with those of miglitol.
\end{abstract}

Methods: This study was a 12-week, open-label, prospective, randomized, parallel-group comparison trial. Fifty-two patients with type 2 diabetes who aged 20 - 70 years with a low-density lipoprotein cholesterol (LDL-C) level of over $120 \mathrm{mg} / \mathrm{dL}$, and with no history of treatment with antihyperlipidemic drugs were enrolled. Patients were randomly assigned to either the anagliptin group or miglitol group. The $100 \mathrm{mg}$ of anagliptin was administered twice a day for the anagliptin group and $50 \mathrm{mg}$ of miglitol was administered thrice a day for miglitol group. The changes in lipids, cholesterol synthesis, and absorption markers were evaluated after 12 weeks.

Results: Fifty-two participants were initially enrolled in the trial, and 47 of them completed the protocol. There was no significant difference in LDL-C, cholesterol synthesis, and the absorption markers between anagliptin and miglitol groups.

Conclusions: Anagliptin and miglitol are similarly effective on lipid and glycemic control.

Keywords: Anagliptin; Miglitol; Type 2 diabetes; Lipids

Manuscript submitted January 8, 2020, accepted January 20, 2020

aDepartment of Endocrinology and Metabolism, Yokohama City University Graduate School of Medicine, Yokohama, Japan

bDepartment of Endocrinology, Diabetes and Metabolism, Yokosuka Kyosai Hospital, Yokosuka, Kanagawa, Japan

'Internal Medicine, Kanagawa Dental University, Yokosuka, Kanagawa, Japan ${ }^{\mathrm{d} C}$ Corresponding Author: Yasuo Terauchi, Department of Endocrinology and Metabolism, Yokohama City University Graduate School of Medicine, Fukuura 3-9, Kanazawa-ku, Yokohama 236-0004, Japan.

Email: terauchi@yokohama-cu.ac.jp

doi: https://doi.org/10.14740/jocmr4084

\section{Introduction}

The number of patients with type 2 diabetes has increased worldwide. Because patients with type 2 diabetes often have dyslipidemia, it is important to normalize their lipid levels in order to prevent cardiovascular diseases [1-3].

Postprandial glucose excursion is correlated with an increase in cardiovascular dysfunction $[4,5]$. Dipeptidyl peptidase-4 (DPP-4) inhibitors and alpha-glucosidase inhibitors $(\alpha$-GIs) are effective in reducing the postprandial glucose levels. Seven types of DPP-4 inhibitors, that are taken daily, are available in Japan.

Takihata et al reported that the administration of sitagliptin, but not pioglitazone, decreased low-density lipoprotein cholesterol (LDL-C) and high-density lipoprotein cholesterol (HDL-C) in Japanese patients with type 2 diabetes [6]. Chiba et al also reported that anagliptin significantly reduced total cholesterol (TC) (i.e. LDL-C and HDL-C levels) in patients with type 2 diabetes [7]. Of note, a meta-analysis of DPP-4 inhibitors suggested that treatment with DPP-4 inhibitors was also associated with a significant reduction in TC [8]. Recently, we reported that lathosterol, a cholesterol synthesis marker, was suppressed after 1 month of treatment with anagliptin, a DPP-4 inhibitor [9]. However, this investigation was observational, and the duration of treatment was short. Therefore, in this study, we administered either anagliptin or miglitol, which are $\alpha$-GIs, for 3 months in patients with type 2 diabetes and compared lipid-lowering effects of anagliptin with those of miglitol.

\section{Materials and Methods}

\section{Subjects}

This study was approved by the Institutional Ethics Review Committee of Yokohama City University Hospital, and the protocol was registered in the University Hospital Medical Information Network (UMIN) Clinical Trial Registry as UMIN000021494. Written informed consent was obtained from each of the patients before the start of the study.

As the desired target for the primary prevention of is- 


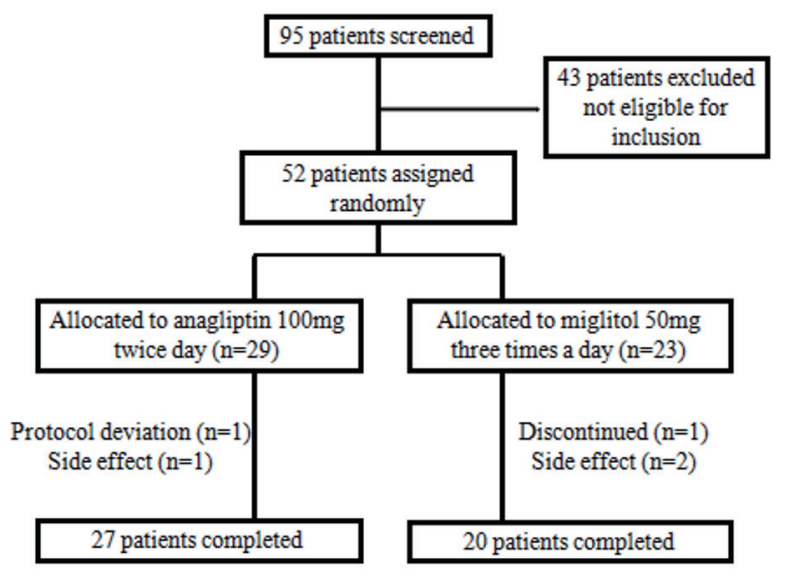

Figure 1. Schematic presentation of this study.

chemic heart diseases in Japanese patients with type 2 diabetes was LDL-C level of $<120 \mathrm{mg} / \mathrm{dL}$, we enrolled male and female patients aged 20 - 75 years with type 2 diabetes LDL-C level of $120 \mathrm{mg} / \mathrm{dL}$ and over and without any prior treatment with DPP-4 inhibitors, $\alpha$-GIs and cholesterol-lowering drugs. Patients were prohibited from receiving another anti-diabetic drug during the study.

\section{Study design}

The study was an open-label, randomized, parallel-group clinical trial. Patients were recruited from March 2016 to November 2018 and randomly assigned (1:1) to either the anagliptin group or the miglitol group (Fig. 1). Patients who were assigned to the anagliptin group took $100 \mathrm{mg}$ tablets twice a day before breakfast and dinner, and patients who were assigned to the miglitol group took $50 \mathrm{mg}$ tablet three times a day before every meal. Patients were asked to visit the hospital at $0,4 \pm$ 1 , and $12 \pm 2$ weeks.

We instructed the patients not to change their daily routine including their food intake and exercise habits for 12 weeks. Patients were asked every day whether they have taken the medicine and were asked to write any side effects in a diary. The results of laboratory data before and after treatment were explained to the patients only after the end of intervention to prevent any changes in diet or exercise based on the knowledge of the laboratory results. A meal tolerance test was conducted before the treatment and 12 weeks after the treatment. All the subjects received a standard breakfast (773 kcal; protein: 27.0 g; fat: $20.3 \mathrm{~g}$; carbohydrate: $121.5 \mathrm{~g}$ ). For the study, the subjects were requested to fast for at least $12 \mathrm{~h}$. Blood samples were collected at 0,60 , and 120 min after breakfast. Blood test was also conducted at 4 weeks after the treatment to check adverse effects of drugs.

\section{Endpoints}

The primary end-point was a difference in LDL-C at 12 weeks between the anagliptin group and the miglitol group. The secondary end-points were differences in TC, HDL-C, nonHDL-C, triglyceride (TG), lathosterol, sitosterol, campesterol, cholestanol, malondialdehyde-modified LDL-C (MDA-LDLC), small dense LDL-C (sd-LDL-C), apolipoprotein B-48 (apoB-48), apoB-100 and hemoglobin A1c (HbAlc) at 12 weeks between the two groups. Lathosterol, sitosterol, campesterol, cholestanol and apoB-48 were measured at SRL Co. (Tokyo, Japan). Cholesterol synthesis and absorption markers were measured using gas chromatography. ApoB-100 was measured using an ELISA kit (Millipore Corp, MA, USA) in our laboratory. Remaining factors were measured in the clinical laboratory of Yokohama City University Hospital.

\section{Statistical analysis}

Similar to our previous study on lipid-lowering effects of anagliptin in 30 outpatients with type 2 diabetes [10], the sample size in each group in this study also included 30 patients (total 60 patients). All statistical factors were decided prior to the study and all the statistical analyses were conducted and monitored by EMI Inc. (Osaka, Japan). Data were expressed as mean \pm standard deviation (SD). Sex ratio/distribution was compared by $\chi^{2}$ test and age. Duration of diabetes and the percentage of patients who did not take any medicine were analyzed by Student's $t$-test. Data between baseline and week 4 in each group were compared by paired $t$-test. Data from the anagliptin group and miglitol group were compared at 12 weeks using a linear mixed model. Differences were considered significant at $\mathrm{P}<0.05$.

\section{Results}

\section{Baseline clinical characteristics}

Table 1 shows the baseline characteristics of patients. Out of 52 patients, 29 patients were assigned to the anagliptin group and 23 patients to the miglitol group, and 27 and 20 of these patients completed the study, respectively. There were no statistically significant differences between the groups with respect to baseline patient characteristics, including the age, sex and the duration of diabetes.

\section{Changes in serum lipid profiles}

In the anagliptin group, $\mathrm{HbA} 1 \mathrm{c}, \mathrm{HDL}-\mathrm{C}$, and non HDL-C were decreased significantly during the 12 -week treatment. However, no significant change was observed in lathosterol, campesterol, sitosterol, and cholestanol. In the anagliptin group, TC was increased by the treatment but the difference was not statistically significant $(P=0.0929)$. In the miglitol group, $\mathrm{HbA1c}$, campesterol and sitosterol decreased significantly by the end of the 12-week treatment. This group also showed an increase in lathosterol. However, this value is not statistically significant $(P=0.0599)$. In conclusion, no significant differ- 
Table 1. Patient Characteristics

\begin{tabular}{llll} 
& Anagliptin group $(\mathbf{n}=\mathbf{2 9})$ & Miglitol group $(\mathbf{n}=\mathbf{2 3})$ & P \\
\hline Sex ratio/distribution (men/women) & $23 / 6$ & $19 / 4$ & 1.0000 \\
Age (years) & $53.7 \pm 8.5$ & $56.3 \pm 8.4$ & 0.2865 \\
Duration of diabetes (years) & $6.5 \pm 7.9$ & $3.6 \pm 3.2$ & 0.0789 \\
Body weight $(\mathrm{kg})$ & $77.0 \pm 17.1$ & $75.6 \pm 13.7$ & $167.8 \pm 8.1$ \\
Height $(\mathrm{cm})$ & $165.6 \pm 7.0$ & $26.8 \pm 4.3$ \\
Body mass index $\left(\mathrm{kg} / \mathrm{m}^{2}\right)$ & $27.9 \pm 4.6$ & & \\
\hline
\end{tabular}

ences in HbA1c, LDL-C, TC, HDL-C, TG, lathosterol, campesterol, sitosterol, cholestanol, apoB-48, apoB-100, sd-LDL, and MDA-LDL were observed between the anagliptin group and miglitol group (Table 2).

Regarding the meal tolerance test, TG and apoB-48 levels decreased at $60 \mathrm{~min}$ in the miglitol group, but there were no significant changes in TG and apoB-48 after 12 weeks of treatment with anagliptin or miglitol (Fig. 2).

\section{Adverse effects}

Adverse effects are described in Table 3. Gastrointestinal symptoms were observed in the anagliptin (three patients, 11.1\%) and miglitol groups (15 patients, $75.0 \%$ ), respectively. None of them discontinued. One case of drug eruption was observed in the anagliptin group and two cases of serious weight loss were observed in the miglitol group. Both patients were not given more medicine. No severe cases of hypoglycemia were observed in either group during the trial. As shown in Table 4, there was no significant difference in the rate of forgetfulness about taking the drugs between the two groups $(1.6 \pm 3.5 \%$ vs. $3.4 \pm 4.6 \%, \mathrm{P}=0.1184)$.

\section{Discussion}

The most important finding of this study was that there was no significant difference in LDL-C, cholesterol synthesis, and absorption marker between the anagliptin and miglitol groups. In the anagliptin group, HDL-C, non HDL-C were decreased significantly by 3 -month treatment. In contrast, in the miglitol group, while non HDL-C was not decreased significantly, campesterol and sitosterol were increased significantly by 3-month treatment, and lathosterol tends to decrease by this treatment, although statistically, the difference is not significant.

DPP-4 inhibitors have been reported to decrease LDL-C and TC levels [6-8]. It was reported that anagliptin was superior to sitagliptin in lowering LDL-C in the patients with type 2 diabetes in a randomized clinical trial [10]. We here evaluated the lipid-lowering effects of anagliptin in Japanese patients with type 2 diabetes in randomized clinical trial by comparing it with miglitol, which improved postprandial plasma glucose similarly. Previously, we administered anagliptin in patients with type 2 diabetes, whose LDL-C levels were over $120 \mathrm{mg} /$
$\mathrm{dL}$, and who were not on any medications [9]. After 1 month, lathosterol was decreased significantly by anagliptin treatment. In this study, we could not observe a significant decrease in lathosterol, and this may be due to the differences in baseline LDL-C, number of subjects, duration of treatment, and/or weak lipid-lowering effect of anagliptin.

Several reports have indicated that cholesterol metabolism can be improved through DPP-4 inhibitors in animal models. Vildagliptin decreased expressions of the genes involved in hepatic cholesterol biosynthesis, such as phosphomevalonate kinase (PMVK), acyl-coenzyme A dehydrogenase medium chain (ACADM), diphosphomevalonate decarboxylase (MVD), and acyl-CoA synthetase (ACSL1) [11]. Yano et al reported that sterol regulatory element-binding protein-2 (SREBP2) mRNA expression level was significantly decreased at night with anagliptin treatment in LDL receptordeficient mice [12]. These results suggest the down-regulation of lipid synthesis by liver. Anagliptin significantly suppressed SREBP activity in HepG2 cells. Goto et al also reported that anagliptin improved hypercholesterolemia in apoE-deficient mice by inhibiting intestinal cholesterol transport [13]. Thus, in animal model studies, DPP-4 inhibitor decreases cholesterol synthesis and absorption. However, the lipid-lowering effect of DPP-4 inhibitors, including anagliptin, is small compared with statins in humans. Therefore, it is difficult to investigate the mechanisms of anagliptin-induced alterations in lipid metabolism by checking cholesterol synthesis and absorption markers.

Regarding the effect of miglitol on lipid metabolism, it was reported that miglitol treatment decreased LDL-C in subjects with metabolic syndrome [14]. In our study, LDL-C levels were not decreased significantly. Fecal concentration of campesterol was reported to be decreased by acarbose in healthy subjects [15]. This result suggests that $\alpha$-GI increases absorption of campesterol and may be consistent with our results, which indicates that miglitol treatment leads to lowered campesterol levels. Thus, lathosterol was decreased for compensation. Further investigation is needed for this effect.

We previously investigated the improvement of drug adherence in patients who had taken miglitol [16]. While miglitol is administered to patients three times a day, anagliptin is administered twice a day in this study. As shown in Table 4, there was no significant difference of drug adherence between anagliptin and miglitol groups, but this may be due to the small sample size. It was previously published that increased number of drug intake worsens the drug adherence [17]. Regarding 


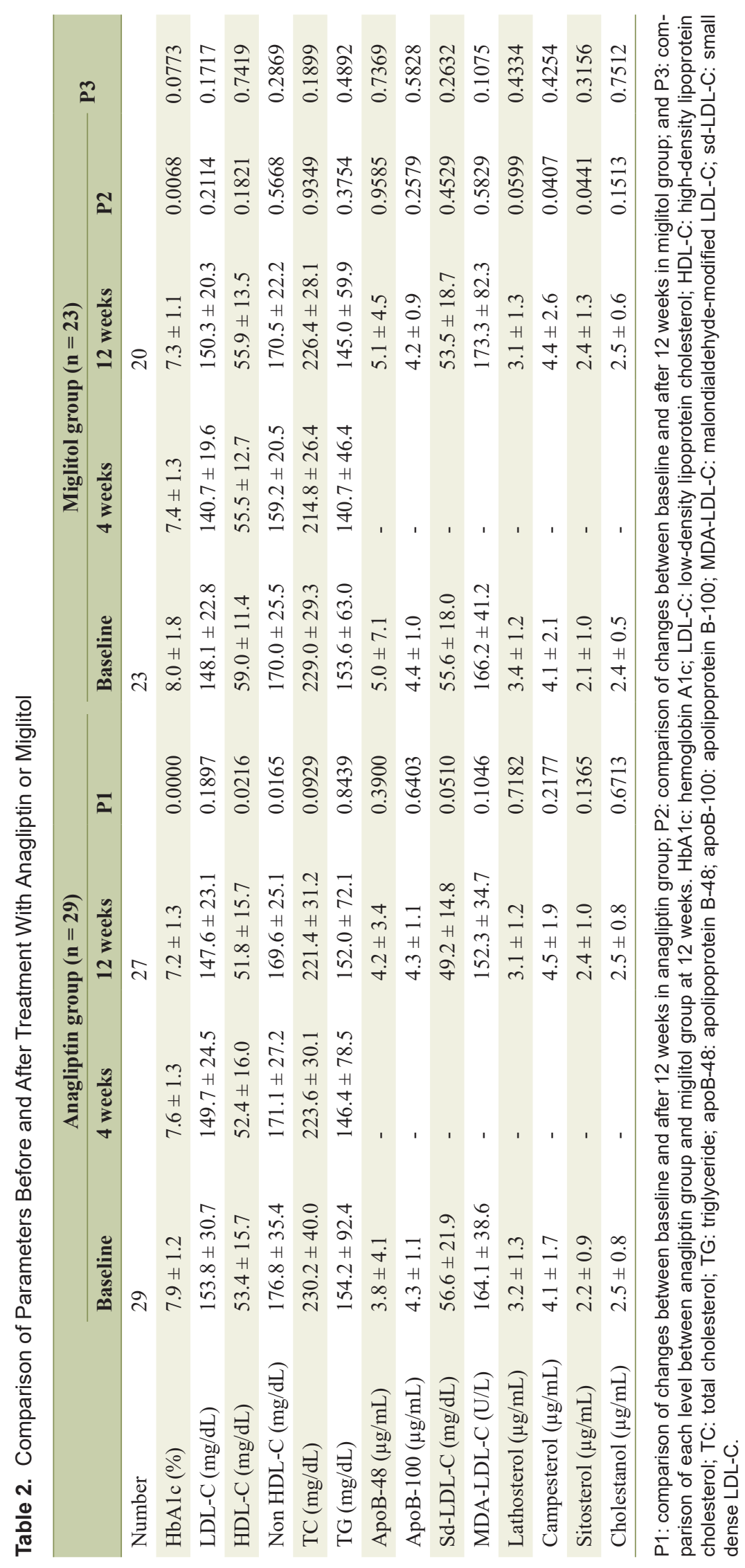



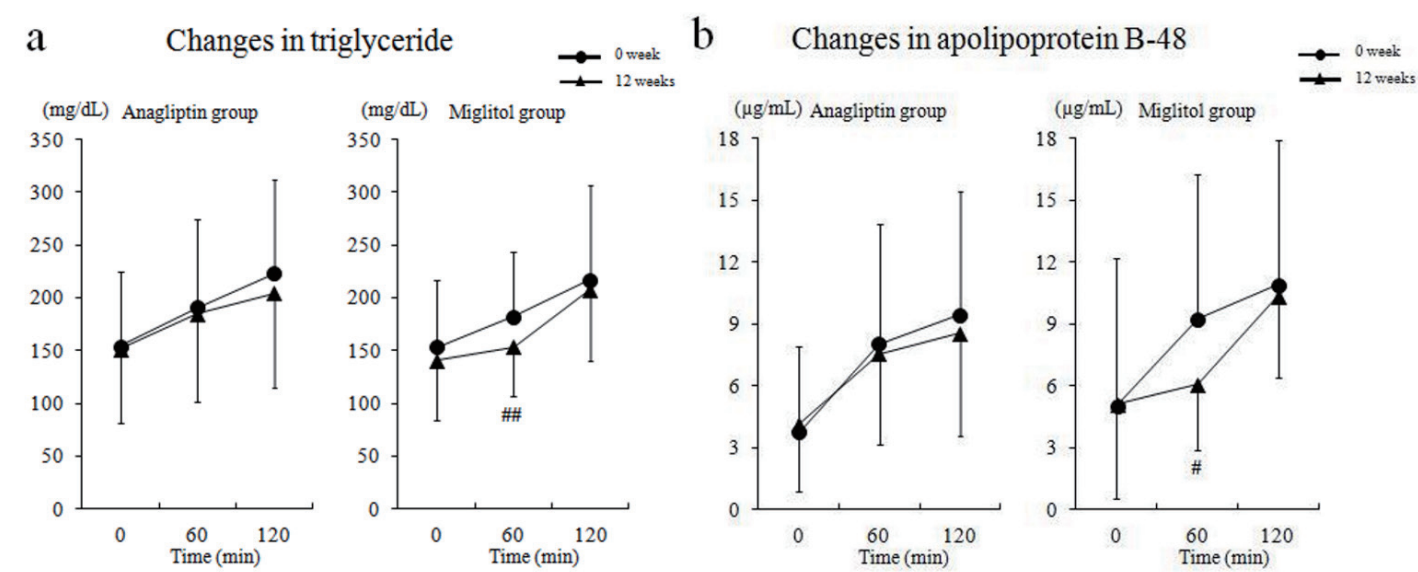

Figure 2. Serum triglyceride and apolipoprotein B-48 levels by meal tolerance test before and after treatment. (a) Changes in triglyceride, and (b) changes in apolipoprotein B-48. \# P < 0.05; \#\# P 0.01 compared with baseline (0 week).

drug adherence and effect on lipids, anagliptin will be administered to patients with postprandial hyperglycemia and high non HDL-C levels, rather than miglitol.

Chiba et al reported that anagliptin treatment decreased LDL-C more in cases with initial high level of LDL-C $(>120)$ than in those with an initial low level of LDL-C $(<120)$ [7]. Therefore, we will investigate lipid-lowering effect of anagliptin according to the baseline LDL-C along with cholesterol synthesis and absorption markers in the post hoc analysis.

The present study had several limitations. We included patients without history of treatment with DPP-4 inhibitors and $\alpha$-GIs or anti-hyperlipidemic drugs to evaluate the effect of anagliptin on lipid profile. Therefore, we could not include a sufficient number of patients. In addition, this study had an open-label design and the duration of anagliptin treatment was short. Therefore, a larger-scale, double-blinded study for a longer period of treatment is needed in the future.

In conclusion, anagliptin and miglitol are similarly effective on lipid and glycemic control.

\section{Acknowledgments}

We appreciate Noriko Akiyama and Takuro Sada for secre- tarial assistance.

\section{Financial Disclosure}

This study was funded by Sanwa Kagaku Kenkyusho Co., Ltd.

\section{Conflict of Interest}

YT has received honoraria for lectures from MSD K.K.; Ono Pharmaceutical Co., Ltd; Nippon Boehringer Ingelheim Co., Ltd; Novartis Pharma K.K.; Takeda Pharmaceutical Co., Ltd; Mitsubishi Tanabe Pharma Corp.; Daiichi Sankyo Co., Ltd; Sanwa Kagaku Kenkyusho Co., Ltd; Kowa Pharmaceutical Co., Ltd; Novo Nordisk Pharma Ltd; Eli Lilly Japan K.K.; Sanofi K.K.; Shionogi \& Co., Ltd; Bayer Yakuhin, Ltd; and AstraZeneca K.K. and has obtained research support from MSD K.K.; Ono Pharmaceutical Co., Ltd; Nippon Boehringer Ingelheim Co., Ltd; Novartis Pharma K.K.; Takeda Pharmaceutical Co., Ltd; Mitsubishi Tanabe Pharma Corp.; Daiichi Sankyo Co., Ltd; Sanwa Kagaku Kenkyusho Co., Ltd; Novo Nordisk Pharma Ltd; Eli Lilly Japan K.K.; Sanofi K.K.; Dainippon Sumitomo Pharma Co., Ltd; Shionogi \& Co., Ltd;

Table 3. Adverse Effects

\begin{tabular}{lll}
\hline Adverse effects & Anagliptin group & Miglitol group \\
\hline Gastrointestinal symptoms & 3 & 15 \\
Drug eruption & 1 & \\
Liver dysfunction & 1 & 2 \\
Weight loss & & 2 \\
\hline
\end{tabular}

Values are $\mathrm{n}$.

Table 4. Medication Adherence

\begin{tabular}{llll} 
Medication adherence & Anagliptin group & Miglitol group & P \\
\hline Percentage forgetting to take oral medication & $1.58 \pm 3.46$ & $3.36 \pm 4.59$ & 0.1184 \\
\hline
\end{tabular}


Bayer Yakuhin, Ltd; Astellas Pharma, Inc.; Pfizer Japan, Inc.; and AstraZeneca K.K. KA has obtained research support from Sanwa Kagaku Kenkyusho Co., Ltd. TI and YK declare that he have no conflicts of interest.

\section{Informed Consent}

Written informed consent was obtained from each of the patients before the start of the study.

\section{Author Contributions}

TI, KA, YK and YT designed this research. TI and KA collected the laboratory data. TI, KA and YT wrote the manuscript.

\section{References}

1. Colhoun HM, Betteridge DJ, Durrington PN, Hitman GA, Neil HA, Livingstone SJ, Thomason MJ, et al. Primary prevention of cardiovascular disease with atorvastatin in type 2 diabetes in the Collaborative Atorvastatin Diabetes Study (CARDS): multicentre randomised placebo-controlled trial. Lancet. 2004;364(9435):685-696.

2. Collins R, Armitage J, Parish S, Sleigh P, Peto R, Heart Protection Study Collaborative G. MRC/BHF Heart Protection Study of cholesterol-lowering with simvastatin in 5963 people with diabetes: a randomised placebo-controlled trial. Lancet. 2003;361(9374):2005-2016.

3. Tajima N, Kurata H, Nakaya N, Mizuno K, Ohashi Y, Kushiro T, Teramoto T, et al. Pravastatin reduces the risk for cardiovascular disease in Japanese hypercholesterolemic patients with impaired fasting glucose or diabetes: diabetes subanalysis of the Management of Elevated Cholesterol in the Primary Prevention Group of Adult Japanese (MEGA) Study. Atherosclerosis. 2008;199(2):455-462.

4. Glucose tolerance and mortality: comparison of WHO and American Diabetes Association diagnostic criteria. The DECODE study group. European Diabetes Epidemiology Group. Diabetes Epidemiology: Collaborative analysis Of Diagnostic criteria in Europe. Lancet. 1999;354(9179):617-621.

5. Group DS, International Diabetes Epidemiology G. Cardiovascular risk profile assessment in glucose-intolerant Asian individuals - an evaluation of the World Health Organization two-step strategy: the DECODA Study (Diabetes Epidemiology: Collaborative Analysis of Diagnostic Criteria in Asia). Diabet Med. 2002;19(7):549-557.

6. Takihata M, Nakamura A, Tajima K, Inazumi T, Komatsu Y, Tamura H, Yamazaki S, et al. Comparative study of sitagliptin with pioglitazone in Japanese type 2 diabetic patients: the COMPASS randomized controlled trial. Diabetes Obes Metab. 2013;15(5):455-462.

7. Chiba Y, Yamakawa T, Tsuchiya H, Oba M, Suzuki D, Danno H, Takatsuka Y, et al. Effect of anagliptin on glycemic and lipid profile in patients with type 2 diabetes mellitus. J Clin Med Res. 2018;10(8):648-656.

8. Monami M, Lamanna C, Desideri CM, Mannucci E. DPP-4 inhibitors and lipids: systematic review and metaanalysis. Adv Ther. 2012;29(1):14-25.

9. Aoki K, Ijima T, Kamiyama H, Kamiko K, Terauchi Y. Anagliptin decreases serum lathosterol level in patients with type 2 diabetes: a pilot study. Expert Opin Pharmacother. 2015;16(12):1749-1754.

10. Morimoto T, Sakuma I, Sakuma M, Tokushige A, Natsuaki M, Asahi T, Shimabukuro M, et al. Randomized Evaluation of Anagliptin vs Sitagliptin On low-density lipoproteiN cholesterol in diabetes (REASON) Trial: A 52-week, open-label, randomized clinical trial. Sci Rep. 2019;9(1):8537.

11. Flock G, Baggio LL, Longuet C, Drucker DJ. Incretin receptors for glucagon-like peptide 1 and glucose-dependent insulinotropic polypeptide are essential for the sustained metabolic actions of vildagliptin in mice. Diabetes. 2007;56(12):3006-3013.

12. Yano W, Inoue N, Ito S, Itou T, Yasumura M, Yoshinaka $\mathrm{Y}$, Hagita S, et al. Mechanism of lipid-lowering action of the dipeptidyl peptidase-4 inhibitor, anagliptin, in lowdensity lipoprotein receptor-deficient mice. J Diabetes Investig. 2017;8(2):155-160.

13. Goto M, Furuta S, Yamashita S, Hashimoto H, Yano W, Inoue N, Kato N, et al. Dipeptidyl peptidase 4 inhibitor anagliptin ameliorates hypercholesterolemia in hypercholesterolemic mice through inhibition of intestinal cholesterol transport. J Diabetes Investig. 2018;9(6):1261-1269.

14. Shimabukuro M, Higa M, Yamakawa K, Masuzaki H, Sata M. Miglitol, alpha-glycosidase inhibitor, reduces visceral fat accumulation and cardiovascular risk factors in subjects with the metabolic syndrome: a randomized comparable study. Int J Cardiol. 2013;167(5):2108-2113.

15. Bartram HP, Scheppach W, Heid C, Fabian C, Kasper H. Effect of starch malabsorption on fecal bile acids and neutral sterols in humans: possible implications for colonic carcinogenesis. Cancer Res. 1991;51(16):4238-4242.

16. Aoki K, Kamiyama H, Yoshimura K, Shibuya M, Masuda $\mathrm{K}$, Terauchi Y. Miglitol administered before breakfast increased plasma active glucagon-like peptide-1 (GLP-1) levels after lunch in patients with type 2 diabetes treated with sitagliptin. Acta Diabetol. 2012;49(3):225-230.

17. Claxton AJ, Cramer J, Pierce C. A systematic review of the associations between dose regimens and medication compliance. Clin Ther. 2001;23(8):1296-1310. 